\title{
Current and Future Challenges In Drug Therapeutics for Sars-Cov-2 Infection in Covid-19 Pandemic
}

\author{
Nitin Saksena* \\ Epigenes Australia Pty Ltd, Australia \\ *Corresponding author: Nitin Saksena, Epigenes Australia Pty Ltd., Melbourne, Australia. \\ To Cite This Article: Nitin Saksena.Current and Future Challenges In Drug Therapeutics for Sars-Cov-2 Infection in Covid-19 Pandemic. 2020 - 9(2). \\ AJBSR.MS.ID.001373. DOI: 10.34297/AJBSR.2020.09.001374.
}

Received: 盋May 12, 2020; Published: 筒 June 18, 2020

Keywords: Pandemic, COVID-19, SARS-CoV-2, Therapeutics, Vaccines, Antivirals

\section{Introduction}

Pneumonia of unknown cause detected in Wuhan; China was first reported to the WHO Country Office in China on December 31 2019. Later, the causative agent of which was identified to be Severe acute respiratory syndrome coronavirus 2 (SARS-CoV-2). On March 11,2020 , the pandemic caused by the SARS-CoV-2 was termed as COVID-19 by the World Health Organization (WHO), with the disease touching practically every country in the world. The SARSCoV-2 infection is, although characterized by flu-like symptoms, the fast progression is attributed to the acute respiratory distress syndrome (ARDS) [1] or myocarditis [2]. Many other manifestations of the disease have been described in different patient groups across different countries. As of June 18, 2020 the total infections globally stand at 8,242, 999 million people, with 445, 535 deaths, $4,530,266$ recoveries. The take-home message is that despite the severity, there are more recoveries.

As the researchers and pharmaceutical companies race to develop vaccines and antivirals, there is a lack of approved treatments for Coronavirus infections. Noteworthy is that most drugs or vaccine approaches that are touted to be useful have not been created taking SARS- CoV-2 in mind, but are being repurposed because of urgency. As a consequence, there is an unmet and urgent need for effective therapeutics, specifically against SARS-CoV-19 (WHO update, May 2020) [3].

This mini-review provides a snapshot of current and future challenges with drug treatments for SARS-CoV-2. Although new vaccines and biologics (monoclonal antibodies) are being devel oped and trailed, it is outside the scope of this review to provide commentary.

\section{Biology of Coronaviruses}

SARS-CoV-2 is a non-segmented, enveloped positive-sense RNA virus belonging to the family Coronaviridae. Although since 2003, we have seen the emergence of SAS-CoV-1, MERS, and now SARSCoV-2, there are already seven coronaviruses that are known to cause infections in humans (NL63, OC43, HKU1, 229E, SARS-CoV-1, MERS-CoV, SARS-CoV-1 and SARS-CoV-2) [2].

The SARS-CoV-2 sequence appears to be closely related to that of SARS-CoV-1 that caused the SARS epidemic in 2003. Thus, what we know of SARS-CoV-2 is primarily extrapolated from SARS-CoV-1 and MERS as all three viruses cause severe respiratory disease in humans. But it is believed that with varied manifestations, not previously seen in SARS-CoV-1 and MERS, the SARS-CoV-2 is highly pathogenic, especially among the older age categories and people with comorbidities such as smoking, diabetes, cardiovascular ailments, and weaker immunity.

To put it into perspective, the World Health Organization warned in a statement released on May 11, 2020, that a review of studies by public health experts convened by the WHO last month found that smokers are "more likely to develop severe disease with COVID-19, compared to non-smokers." It is known that smoking impairs lung function and makes it more difficult for the body to fight off coronaviruses and other diseases, and many other respiratory infections and increases the severity of respiratory diseases. According to the WHO, "tobacco is also a major risk factor for non communicable diseases like cardiovascular disease, cancer, respiratory disease and diabetes which put people with these conditions at higher risk for developing severe illness when 
affected by COVID-19," Direct deaths per year as a result of tobacco use are $>7$ million people.

Coronaviruses generally spread from human-to-human through respiratory droplets, and this spread is exacerbated by population density and poor hygiene. SARS-CoV-1 and SARS-CoV-2 enter the host cells by using spike protein on the surface of the viral capsid, which binds to angiotensin-converting enzyme II (ACE2) on type II alveolar cells leading to fusion of the viral and host cell membranes for the transfer of viral RNA into the host cytoplasm [4]. Once gaining entry into the cells using the angiotensin receptor, the viral replication causes the release of newly formed virus particles or virions, where each of these steps in the viral life cycle presents a target for subduing the virus and disrupt the viral life cycle. Strategies capable of disrupting S protein interaction with ACE2 are thought to carry significant therapeutic value. It is also because the binding affinity of SARS-CoV-2 S protein to ACE2 is 10-20-fold higher than for the S protein of SARS-CoV, and this particular aspect is attributed to higher infectious potential and contagiousness of SARS-CoV-2 as compared to SARS-CoV $[4,5]$.

\section{Current therapeutics and future challenges with drugs}

\section{Viral entry inhibitors}

\section{a. Intuitive use of angiotensin-converting enzyme inhibitors or ACE2 receptor blockers in SARS- CoV-2 infection}

As the virus uses the ACE2 receptor to gain entry into the host cell, it is intuitive to use receptor blockers that can curtail viral entry. As per the WHO update on May 2020, there is a significant concern, based on considerations of biological plausibility and the observation on the overrepresentation of patients with hypertension and other cardiovascular comorbidities among patients with COVID-19 who have poor outcomes, that angiotensinconverting enzyme inhibitors (ACE inhibitors) and angiotensin receptor blockers (ARBs) enhance susceptibility to coronavirus SARS CoV-2 raising the likelihood of severe COVID-19 illness [5-7]. There are pre- existing angiotensin receptor blockers (ARBs such as losartan, valsartan, and telmisartan) used in humans do present a novel therapeutic approach to block the attachment of SARSCoV-2 spike protein to ACE2-expressing cells, thereby inhibiting the infection of host cells. However, their effects should be weighed with caution as they are not antiviral agents. There is a development of a whole range of small molecule ACE2 inhibitors from chemical and photochemical sources [5]. Millions of people around the world are on treatment with ACE- Is and ARBs for hypertension, heart failure, coronary artery disease, or kidney disease. There is low-certainty evidence that patients on long-term therapy with ACE inhibitors or ARBs are not at higher risk of poor outcomes from COVID-19, according to the studies done in Europe and China. Further, at this time, no direct evidence is available that can point to a clear-cut efficacy of or any clinical impact of ACE inhibitors on in SARS-CoV-2 infected patients. Hypertension, diabetes, and cerebrovascular disease have been identified for poor prognosis for SARS-CoV-2 treatment in a retrospective study from Wuhan, China [8-12].

\section{b. Chloroquine and Hydroxychloroquine: an anti-malarial drug repurposed}

Also called 4-aminoquinoline is primarily an antimalarial drug. It is being repurposed to treat SARS-CoV-2 infection as mechanistically it can alter the glycosylation of ACE2, thereby decreasing the binding between the viral spike protein and the receptor ACE2 on the host cell, subsequently inhibiting viral entry into the host cell in vitro [13]. Moreover, hydroxychloroquine is also known to inhibit the Toll-like receptor (TLR) pathway, which is intrinsically involved in pro-inflammatory cytokine signaling [14], thereby reducing the inflammation. Reducing inflammation mainly in the lung, resulting from cytokine storm, has been thought to be a key aspect in controlling the disease.

Chloroquine inhibits SARS-CoV-2 infectivity [14], along with evidence showing that it also inhibits SARS-CoV-2 entry [15]. 500 mg Chloroquine twice a day for 10 days was prescribed in China SARS-CoV-2 infection, but further data on full efficacy are not available [16]. These beneficial effects of Chloroquine, which still needs proof in patients, come with toxicity previously described by many studies. These toxic effects ranging from dysrhythmias, hypotension, neurologic effects, oxidative stress, causing hemolysis, and potassium channel blockade have been seen at doses higher than 5 grams [17-19]. Hydroxychloroquine is less toxic than Chloroquine, but the exact mechanism remains poorly understood. Its toxic effects, which overlap with Chloroquine, start at 4grams.

Overall, the data on the efficacy of hydroxychloroquine in treating COVID-19 is minimal, apart from anecdotal or sporadic success in treating SARS-CoV-2 infection [20]. Thus, caution is needed in using this drug, keeping in mind the com orbidities patients may have. SARS-CoV-2 has an affliction of comorbidities, as has been seen during the COVID-19 pandemic in China, Spain, Italy, Iran, and the USA (WHO update 2020).

\section{Viral Replication Inhibitors}

All RNA viruses depend on the host cellular machinery to translate viral RNA into proteins. The RNA viruses do this seamlessly in achieving virion production and synthesis of all proteins involved in infection, such as the Spike and the Capsid proteins. Various drug categories such as Nucleotide analogs and protease inhibitors previously used for the Ebola virus and HIV are being repurposed for treating SARS-CoV-2. 


\section{Nucleoside Analogs}

\section{Remdesivir}

Remdesivir, an antiviral drug produced by Gilead USA, is a prodrug metabolized to an adenosine nucleoside analog and was used for Ebola and influenza virus treatment. It also has demonstrated in vitro efficacy against SARS-CoV-2, as shown recently [21], and has shown efficacy by shortening the duration of illness in Phase 3 human trial. There is still a significant need for agents that modulate the host immune response, which can make the treatment effective. The optimal duration of Remdesivir treatment is still being studied in ongoing clinical trials. Under the EUA, both 5-day and 10-day treatment durations are suggested, based on the severity of the disease. The authorization is temporary and does not take the place of the formal new drug application submission, review, and approval process. The EUA allows for the distribution and emergency use of remdesivir only for the treatment of COVID-19; remdesivir remains an investigational drug and $\mathrm{h}$ a s not been approved by FDA (https://www. gilead.com/news-and-press/press-room/press-releases/2020/5/ gileads-investigational-antiviral-remdesivir-receives-us-foodand-drug-administration-emergency- use-authorization-for-thetreatment-of-covid19). According to Dr. Steven M. Opal, Infectious Disease Specialist at the Brown University Alpert Medical School of Brown University, remdesivir is not a home-run cure by any means. However, it can be beneficial in the context of better treatment for patients, and a clear understanding is needed on how and when to treat COVID-19 patients, and what other adjunct drugs can provide complete relief. Previous observations on Ebola virus treatment in Africa show that the mortality rate was $18 \%$ among patients receiving invasive ventilation and $5 \%$ among patients not receiving invasive ventilation, suggesting that remdesivir can be a therapeutic option for COVID-19 patients, not on invasive ventilation support. Trials are underway in China, the USA, the Republic of Korea, Singapore, Hong Kong, France, and Taiwan [22].

Favipiravir (6-fluoro-3-hydroxy-2-pyrazinecarboxamide) (by Toyama Chemicals, Japan) is a broad-spectrum antiviral prodrug, which requires in vivo conversion via human hypoxanthine-guanine phosphoribosyltransferase (HGPRT) to an active metabolite to exert an antiviral effect. It is known to potently inhibit the RNAdependent RNA polymerase (RdRP) of RNA viruses by inducing lethal RNA transversion mutations, leading to a non-viable virus phenotype. It is known to inhibit replication of a large number of RNA viruses, including influenza A virus, flavi-, alpha-, filo-, bunya-, arena- and noroviruses, as well as West yellow fever virus, Footand- mouth-disease virus, West Nile virus, and Ebola virus, etc. [23]. Favipiravir does not hold approval in the US for any indication but is approved in Japan and China for the treatment of influenza and novel influenza. It has been approved for experimental use and is in clinical trials in the US, Italy, Japan, and China to treat patients with the novel corona virus.

In the absence of toxicity data on remdesivir, one can only extrapolate drug toxicity from the pre-existing nucleoside analogs used in the treatment of HIV. These nucleoside analogs cause metabolic acidosis, peripheral neuropathy, bone marrow suppression, pancreatitis, and myopathy, with each of these toxicities underpinned into mitochondrial dysfunction, as these analogs in addition to inhibiting viral polymerase, they also inhibit mitochondrial DNA polymerase-gamma, which, consequently, leads to decrease in mitochondrial DNA and proteins [24]. For Favipiravir, repeat-orally administered dose toxicity studies involving dogs, rats, and monkeys, showed adverse effects on hematopoietic tissues such as decreased red blood cell (RBC) production, and increases in liver function parameters such as aspartate aminotransferase (AST), alkaline phosphatase (ALP), alanine aminotransferase (ALT) and total bilirubin, and increased vacuolization in hepatocytes, in addition to testis toxicity (https://www.drugbank.ca/drugs/ DB12466).

\section{Protease inhibitors}

The main feature of the protease inhibitors lies in the prevention of infected cells from stopping the virus from using the host machinery in producing new virions and stopping the viral replication. The viral proteases differ between different viruses due to structural conformation in each virus; therefore, different viral proteases may show differential activity against different kinds of protease inhibitors. The crystal structure of viral proteases is of considerable significance in defining the structural-action relationship (SAR) between the active site in the crystal and the drug. Fortunately, the crystal structure of the SARS-CoV-2 main protease has recently been defined [25], which closely resembles that of SARS-CoV-1 [25], and this may assist in further defining the specificity of either the existing protease inhibitors or designing new ones through SAR analysis.

\section{Lopinavir/ritonavir (KaletraTM): Is combination drug treatment the future?}

Lopinavir (ABT-378) has been used mainly for HIV, and it is a highly potent inhibitor of HIV protease, which plays a significant role in the intracellular HIV assembly. A combination of lopinavir and ritonavir (KaletraTM) is now an established and effective oral drug for the treatment of HIV-infected individuals. The basis for its use in the treatment of SARS emanates from a study conducted in 2003 where lopinavir showed the antiviral property at $4 \mu \mathrm{g} /$ $\mathrm{ml}$ inhibiting the cytopathic effect in a plaque reduction assay in fetal rhesus kidney-4-cells infected with SARS-CoV(HKU-39849 isolate). In this study, newly diagnosed SARS patients infected with SARS-CoV-1 were also treated with the combination of lopinavir (400 mg)/ritonavir (100 mg) orally every 12 hours for 14 days, with patients showing a milder disease course with a considerable 
reduction in viral load and fever by day 21 [26]. Similar results in human trials against MERS were also observed [27].

In contrast, a recent randomized, controlled, and an openlabel trial with 199 hospitalized adult patients in Wuhan/China with certain SARS-CoV-2 infection and pneumonia, the oral administration of twice-daily $400 \mathrm{mg}$ lopinavir and $100 \mathrm{mg}$ ritonavir for 14 days showed that the treatment with lopinavir/ritonavir combination was not associated with a reduction of viral RNA loads or duration of viral RNA detectability as opposed to 100 controls [28]. These observations require further study, and clinical trials with lopinavir/ritonavir in COVID-19 patients have recently been initiated in several countries investigating remdesivir, lopinavir/ ritonavir, and lopinavir/ritonavir plus interferon $\beta$-1a [29].

\section{Umifenovir (ArbidolTM)}

Umifenovir (ArbidolTM), (ethyl-6-bromo-4-[(dimethylamino) methyl]-5-hydroxy-1-methyl-2 [(phenylthio)methyl]-indole-3carboxylate hydrochloride monohydrate), is another protease inhibitor- a small indole-derivate molecule manufactured by JSC Pharmstandard, Russia [105]. Umifenovir blocks viral entry into the host cell by inhibiting membrane fusion of the viral envelope and host cell membrane, thereby preventing host cells from virus infection [30].

In another clinical pilot trial conducted on 36 patients from China with COVID-19 disease, patients received $400 \mathrm{mg}$ umifenovir three times a day for 9 days, against 31 untreated COVID- 19 patients as a control group [31]. Umifenovir showed evidence in reducing viral load as evident by RT-PCR, with decreased mortality ( $0 \%$ vs. $16 \%$ ), as compared to the control group. Further, a retrospective cohort study from a single center in Guangdong, China, 16 patients with COVID-19 disease were enrolled and received $200 \mathrm{mg}$ umifenovir every $8 \mathrm{~h}$ plus lopinavir ( $400 \mathrm{mg}$ )/ritonavir (100 mg) every $12 \mathrm{~h}$ for 5-21 days orally; whereas 17 COVID-19 patients received lopinavir (400 mg)/ritonavir (100 mg) every $12 \mathrm{~h}$ and served as a control group [32]. Following treatment, the detection of SARS-COV-2 was negative in $94 \%$ of the umifenovir- treated patients vs. $53 \%$ in the control group, as evident by RT-PCR assay. Moreover, the chest computed tomography scans also showed improvement in $69 \%$ of the umifenovir-treated patients vs. $29 \%$ in the control group [33]. Because of these clinical results, clinical trials with umifenovir alone or in combination with lopinavir/ritonavir, chloroquine phosphate or carrimycin have been recently initiated in China [34], and it appears that the combination therapy will be the future for yet another RNA virus, and this has already been seen in case of HIV where no single drug was fully effective.

In regards to toxicity, it is very well known for this class of inhibitors as they have been used in HIV treatment. So far, the experience with SARS-CoV-2 treatment does suggest toxicity with lopinavir-ritonavir, mainly at the level of nausea and vomiting, lipodystrophy, as seen before in HIV patients, and mild transaminase elevation across different studies, and the main reason for patient drop-out rates [35].

\section{Conclusions}

Past lessons with most of the drugs listed in this review we already have seen the emergence of drug resistance over time, attribute to viral mutations. In addition, resistance to vaccines has also been shown in seasonal influenza viruses due to less error correction activity of their nucleotide polymerases required in virus replication. In case of flu vaccines which are widely used seasonally and globally, the efficacy of flu vaccines against both influenza A and $B$ viruses is around $40 \%$. Moreover, these viruses can become resistant to anti-influenza drugs, a scenario analogous to HIVan RNA virus. For instance, in the US, out of three neuraminidase inhibitors (NAI) recommended by the CDC (oseltamivir, zanamivir, and peramivir), most circulating influenza viruses appear to be susceptible to the NAI inhibitors, but recent viral strains display significant drug resistance, despite being launched in the same year.

This is a lesson to be learned for SARS-CoV-2 drug treatment, and new direction is needed how we target the virus, whether with small molecules, vaccines, Nucleoside Analogs or immunologic treatments.

\section{Conflicts of Interest}

There are no conflicts to declare.

\section{Acknowledgements}

None

\section{Reference}

1. Yang X, Yu Y, Xu J, Shu H, Xia J, Liu H, et al. (2020) Clinical course and outcomes of critically ill patients with SARS-CoV-2 pneumonia in Wuhan, China: a single-centered, retrospective, observational study. Lancet Respir Med 8(5): 475-481.

2. Mehta P, McAuley DF, Brown M, Sanchez E, Tattersall RS, eta al. (2020) COVID-19: consider cytokine storm syndromes and immunosuppression. Lancet 395(10229): 1033-1034

3. (2020) COVID-19 Coronavirus Pandemic Last updated.

4. Zhou P, Yang X Lou, Wang XG, Hu B, Zhang L, et al. (2020) A pneumonia outbreak associated with a new corona virus of probable bat origin. Nature 579(7798): 270-273.

5. Watkins J (2020) Preventing a covid-19 pandemic. BMJ 368: m810.

6. Wan Y, Shang J, Graham R, Baric RS, Li F (2020) Receptor Recognition by the Novel Coronavirus from Wuhan: an Analysis Based on DecadeLong Structural Studies of SARS Corona virus. Journal of virology 94(7): e00127-20.

7. (2020) Novel Corona virus Pneumonia Emergency Response Epidemiology Team. Vital surveillances: the epidemiological characteristics of an outbreak of 2019 novel corona virus diseases (COVID-19)-China. China CDC Weekly.

8. Rentsch CT, Kidwai-Khan F, Tate JP, et al. (2020) Covid-19 Testing, Hospital Admission, and Intensive Care Among 2,026,227 United States Veterans Aged 54-75 Years.

9. Bean D, Kraljevic Z, Searle T, et al. (2020) Treatment with ACE-inhibitors is associated with less severe disease with SARS-Covid-19 infection in a 
multi-site UK acute Hospital Trust.

10. Liu Y, Huang F, Xu J, et al. (2020) Anti-hypertensive Angiotensin II receptor blockers associated to mitigation of disease severity in elderly COVID-19 patients.

11. Feng Y, Ling Y, Bai T, Huihuang Lin, Jiayang Yan, et al. (2020) COVID-19 with Different Severity: A Multi-center Study of Clinical Features. American Journal of Respiratory and Critical Care Medicine.

12. Meng J, Xiao G, Zhang J, Xing He, Min Ou, et al. (2020) Renin-angiotensin system inhibitors improve the clinical outcomes of COVID-19 patients with hypertension. Emerging Microbes \& Infections 9(1): 757-760.

13. Vincent MJ, Bergeron E, Benjannet S, Erickson BR, Rollin PE, et al. (2005) Chloroquine is a potent inhibitor of SARS coronavirus infection and spread. Virol J 2: 69.

14. Schrezenmeier E, Dörner T (2020) Mechanisms of action of hydroxychloroquine and chloroquine: implications for rheumatology. Nat Rev Rheumatol 16(3): 155-166.

15. Wang M, Cao R, Zhang L, Yang X, Liu J, Xu M, et al. (2020) Remdesivir and chloroquine effectively inhibit the recently emerged novel coronavirus (2019-nCoV) in vitro. Cell Res 30(3): 269-271.

16. Zhonghua Jie He He Hu Xi Za Zhi (2020) Multicenter collaboration group of department of science and technology of Guangdong Province and Health Commission of Guangdong Province for chloroquine in the treatment of novel coronavirus pneumonia. Expert Consensus on chloroquine phosphate for the treatment of novel coronavirus pneumonia 43(0): E019.

17. Riou B, Barriot P, Rimailho A, Baud FJ (1988) Treatment of severe chloroquine poisoning. N Engl J Med 318(1): 1-6.

18. Waldrop T, Alsup D, McLaughlin EC. Fearing coronavirus, Arizona man dies after taking a form of chloroquine used in aquariums.

19. Busari S, Bukola A (2020) Nigeria records chloroquine poisoning after Trump endorses it for coronavirus treatment.

20. Chen Z, Hu J, Zhang Z, Jiang S, Han S, Yan D, et al. (2020) Efficacy of hydroxychloroquine in patients with COVID-19: results of a randomized clinical trial. MedRxiv.

21. Wang M, Cao R, Zhang L, Yang X, Liu J, Xu M, et al. (2020) Remdesivir and chloroquine effectively inhibit the recently emerged novel coronavirus (2019-nCoV) in vitro. Cell Res 30(3): 269-271.

22. McKee DL, Sternberg A, Stange U, Laufer S, Naujokat C (2020) Candidate drugs against SARS-CoV-2 and COVID-19, Pharmacological Research 157: 104859

23. Jin Z, Smith LK, Rajwanshi VK, Kim B, Deval J (2013) The Ambiguous Basepairing and high substrate efficiency of T-705 (Favipiravir) Ribofuranosyl 5 '-triphosphate towards influenza a virus polymerase. PLoS One 8(7): e68347.

24. Michael A Chary, Alexander F Barbuto, Sudeh Izadmehr, Bryan D Hayes, Michele M Burns, et al. (2020) COVID-19: Therapeutics and Their Toxicities. J Med Toxicol 30:1-11.

25. Zhang L, Lin D, Sun X, Curth U, Drosten C, Sauerhering L, et al. (2020) Crystal structure of SARS- $\mathrm{CoV}-2$ main protease provides a basis for design of improved $\alpha$-ketoamide inhibitors. Science 368(6489): 409412 .

26. CM Chu, VCC Cheng, IFN Hung, MML Wong, KH Chan, et al. (2004) Role of lopinavir/ritonavir in the treatment of SARS: initial virological and clinical findings, Thorax 59(3): 252-256.

27. JF Chan, Y Yao, ML Yeung, WDeng, L Bao, L Jia, et al. (2015) Treatment with lopinavir/ritonavir or interferon- $\beta 1 \mathrm{~b}$ improves outcome of MERS$\mathrm{CoV}$ infection in a nonhuman primate model of common marmoset. J Infect Dis 212(12): 1904-1913.

28. B Cao, Y Wang, D Wen, W Liu, J Wang, G Fan, et al. (2020) A trial of lopinavir-ritonavir in adult hospitalized with severe Covid-19, N. Engl J Med.

29. (2020) NCT04315948, Clinical Trials gov.

30. J Blaising, SJ Polyak, EI Pécheur (2014) Arbidol as a broad sprctrum antivital: un update, Antiviral Res 107(2014): 84-94.

31. IA Leneva, RJ Russell, YS Boriskin, AJ Hay AJ (2009) Characteristics of arbidol-resistant mutants of influenza virus: implications for the mechanism of anti-influenza action of arbidol, Antiviral Res 81(2): 132140 .

32. Z Wang, B Yang, Q Li, L Wen, R Zhang (2020) Clinical features of 69 cases with coronavirus disease 2019 in Wuhan, China, Clin Infect Dis 272.

33. L Deng, C Li, Q Zeng, X Liu, X Li, H Zhang, et al., (2020) Arbidol combined with $\mathrm{LPV} / \mathrm{r}$ versus LPV/r alone against corona virus disease 2019: a retrospective cohort study, J Infect S0163-4453(20)30113-4.

34. (2020) NCT04255017, Clinical Trials.gov.

35. Young BE, Ong SWX, Kalimuddin S, Low JG, Tan SY, et al. (2020) Epidemiologic Features and Clinical Course of Patients Infected With 